\title{
Condyle-glenoid fossa relationship after Herbst appliance treatment during two stages of craniofacial skeletal maturation: A retrospective study
}

\author{
Paula Loureiro Cheib Vilefort $^{1}$ | Leticia Orefice Farah ${ }^{2}$ | Henrique Pársia Gontijo ${ }^{1}$ | \\ Alexandre Moro $^{2}$ | Antonio Carlos de Oliveira Ruellas ${ }^{3}$ | Lucia Helena Soares Cevidanes $^{3}$ | \\ Tung Nguyen ${ }^{4}$ (D) | Lorenzo Franchi ${ }^{5}$ (D) | James A. McNamara Jr ${ }^{3}$ | \\ Bernardo Quiroga Souki ${ }^{1}$
}

${ }^{1}$ Graduate Program in Dentistry, Pontifical Catholic University of Minas Gerais, Belo Horizonte, Brazil

${ }^{2}$ Graduate Program in Dentistry, University Positivo, Curitiba, Brazil

${ }^{3}$ Department of Orthodontics and Pediatric Dentistry, School of Dentistry, University of Michigan, Ann Arbor, MI, USA

${ }^{4}$ Department of Orthodontics, School of Dentistry, University of North Carolina, Chapel Hill, NC, USA

${ }^{5}$ School of Dentistry, University of Florence, Florence, Italy

\section{Correspondence}

Bernardo Quiroga Souki, Graduate Program in Dentistry, Pontifical Catholic University of Minas Gerais, Avenida Dom José Gaspar, 500, Coração, Eucarístico, Belo Horizonte, MG 30535-901, Brazil.

Email: souki.bhe@terra.com.br

Funding information

CAPES (Coordenação de Aperfeiçoamento de Pessoal de Nível Superior, Grant/Award Number: \#88881-134753/2016-01

\begin{abstract}
Objectives: To perform a three-dimensional evaluation of the position of the condyles in patients treated with Herbst appliance $(\mathrm{HA})$ in two stages of cervical vertebral maturation.

Setting and sample population: Retrospective case-control study. Pubertal Herbst group (PHG; $n=24$, mean age 14.5 years, CS 3 and CS 4) and pre-pubertal Herbst group (PPHG; $\mathrm{n}=17$, mean age 9.9 years, CS 1 and CS 2) were contrasted with comparison groups of non-orthopaedically treated Class II patients in pubertal (PCG; $\mathrm{n}=17$, mean age 13.9 years) and pre-pubertal maturational stages (PPCG; $n=18$, mean age 10.6 years).

Materials and Methods: Cone-beam computer tomography scans were taken before treatment (TO) and at T1 after 8 to 12 months. Point-to-point measurements of the displacement of the condyles between $\mathrm{TO}$ and $\mathrm{T} 1$, relative to the glenoid fossae, were performed in the $\mathrm{X}, \mathrm{Y}, \mathrm{Z}$ and $3 \mathrm{D}$ perspectives. Qualitative assessments using semitransparent overlays and colour mapping also were produced.

Results: The displacement of the condyles within the glenoid fossae in the treated groups was small $(<0.75 \mathrm{~mm} ; P>.05)$. Relative to the glenoid fossa, condylar position at T1 was similar to T0 in pre-pubertal and pubertal groups $(P>.05)$. Similar condylar rotations from T0 to T1 were observed in Herbst and comparison groups, and no significant difference was found between pre-pubertal and pubertal patients.

Conclusions: Regardless the stage of skeletal maturation, HA treatment did not change the condyle-glenoid fossa relationship.
\end{abstract}

KEYWORDS

Angle Class II, Herbst appliance, imaging, Mandibular condyles, temporomandibular joint 


\section{1 | INTRODUCTION}

Effective correction of Class II malocclusion relies on differential condylar growth that occurs during puberty, ${ }^{1}$ and therefore, it is recommended that orthopaedic treatment with mandibular advancement devices be used during this stage of maturation to obtain optimal skeletal effects. ${ }^{2}$ However, special circumstances such as the increased risk of dental trauma $^{3}$ and psychosocial impairment due to prominent maxillary incisors ${ }^{4}$ might warrant early pre-pubertal correction of Class II malocclusion.

A clinical concern of Herbst appliance (HA) treatment is whether or not the condyles reestablish their centric positions within the glenoid fossa by the end of functional jaw orthopaedic treatment. ${ }^{5,6}$ If condylar growth potentials during pre-pubertal and pubertal stages are different, it follows that positional adaptations within the temporomandibular joint (TMJ) following HA treatment at different stages of maturation also might be different.

To date, most studies on condylar positional changes following HA treatment have been performed using two-dimensional (2D) cephalometric imaging or magnetic resonance imaging (MRI) and/ or computed tomography, still relying on 2D multiplanar assessment of the changes in condylar position. ${ }^{7-17}$ Unfortunately, 2D methods have questionable validity and reproducibility due to differences in magnification, distortion and problems related to patient positioning. Multiplanar topographic assessments provide an improved method relative to $2 \mathrm{D}$ cephalometry, but also have limitations in that anatomic landmarks might not be in the same plane of space, and the three-dimensional (3D) components between two time point records (components $\mathrm{X}, \mathrm{Y}$ and $\mathrm{Z}$ ) might not be measured correctly.

Currently, 3D assessments using cone-beam computer tomography (CBCT) have opened new horizons in the evaluation of positional changes in the condyle. 3D studies of HA therapy, however, are not in full agreement regarding changes in condylar position after treatment. LeCornu et $\mathrm{al}^{18}$ found forward positioning of the condyles after Herbst appliance treatment, while Atresh et $\mathrm{al}^{19}$ found slightly backward positioning. It should be noted that these studies evaluated condylar displacement relative to the anterior cranial base rather than relative to the position of the condyle within the glenoid fossa. Ideally, the centric relationship of the condyle to fossa should be maintained after HA treatment. Further, a systematic review by Al-Saleh and coworkers on this topic found several methodological flaws, recommending additional 3D investigations for a thorough understanding of the fixed appliance's effect on TMJ. ${ }^{20}$

Therefore, the aim of this study was to evaluate condylar position relative to the glenoid fossa after HA treatment, comparing the spatial changes in the condyle of patients treated during pubertal and pre-pubertal stages of skeletal maturation.

\section{2 | MATERIALS AND METHODS}

\section{1 | Study design and sample}

We followed the STROBE statement ${ }^{21}$ for transparent reporting of this observational case-control study. The investigation was approved by the Institutional Review Board of the Pontifical Catholic University of Minas Gerais (number 057992/2016).

This retrospective clinical study was based on a review of 1328 consecutive orthodontic records from two universities databases of patients treated between February 2009 and December 2015 (Pontifical Catholic University of Minas Gerais, Belo Horizonte, Brazil; n = 578; and The Positivo University, Curitiba, Brazil; $n=750$ ). Sample size calculation was performed using $\mathrm{G}^{*}$ Power 3.1 based on the standard deviation of $0.74 \mathrm{~mm}$ reported by Arieta-Miranda et al ${ }^{22}$ relative to the variation in anterior distance of the condyles relative to the glenoid fossa in Class II patients, the primary focus of this investigation. Considering $\alpha$ of $5 \%$ and a power of $95 \%$ to detect condylar positional changes greater than $1 \mathrm{~mm}$ (clinically acceptable difference between groups), a sample size of 16 was needed in each group.

The following inclusion criteria were applied: (a) skeletal Class II malocclusion, characterized by an ANB angle greater than 4 degrees before treatment (TO); (b) dental Class II canine relationship of $4 \mathrm{~mm}$ or more; (c) no previous orthopaedic/orthodontic treatment; (d) no reported sign or symptoms of temporomandibular joint disorder; (e) availability of CBCT scans acquired at $\mathrm{TO}$ and T1; (f) age ranging from 8 years to 16 years at TO; $(\mathrm{g})$ no craniofacial syndrome or deformities; and (h) treatments performed between T0 and T1 scans were either mandibular advancement with HA for the correction of Class II malocclusion or other dental treatments but with no direct impact on the Class II relationship.

The resulting sample of 76 patients consisted of 41 patients in the HA group and 35 patients in the comparison group. The comparison groups ('controls') consisted of patients who required other modalities of dental treatment prior to orthopaedic Class II correction. These conditions included management of impacted teeth, deviations in dental eruption and marsupialization of cysts, all of which had no direct impact on maxillo-mandibular growth. No intermaxillary elastics were used in comparison patients.

The HA group was divided further into a pre-pubertal group (PPHG, $\mathrm{n}=17$, mean age $9.9 \pm 1.9$ years at T0, mean treatment time 12.1 months, cervical vertebral maturational stages CS 1 and CS $2)^{23}$ and a pubertal group ( $\mathrm{PHG}, \mathrm{n}=24$, mean age $14.5 \pm 1.6$ years at TO, mean treatment time 8.4 months, cervical stages CS 3 and CS 4). ${ }^{23}$ The comparison group also was divided into a pre-pubertal group (PPCG, $\mathrm{n}=18$, mean age $10.6 \pm 1.8$ years at T0, mean treatment time 10.2 months, CS1 and CS2) and pubertal group (PCG, $n=17$, mean age $13.9 \pm 1.8$ years at T0, treatment time 10.2 months, CS3 and CS4). The flowchart of the sampling distribution is shown in Figure 1.

All Herbst patients treated with a cantilever HA design received a 'one-step' mandibular activation to obtain molar and canine Class I relationships (Figure $2 \mathrm{~A}-\mathrm{G}$ ). No overcorrection was performed. The mean mandibular advancement immediately after Herbst appliance insertion was $5.5 \mathrm{~mm}$, ranging from $4.0 \mathrm{~mm}$ to $10.0 \mathrm{~mm}$. The duration of treatment followed the protocols of the institution of origin of each patient. The rationale for a more extended period (12 months vs 8 months) for pre-pubertal patients was the slower rate of facial growth during pre-puberty. ${ }^{24}$ 
FIGURE 1 Flowchart of the sampling process [Colour figure can be viewed at wileyonlinelibrary.com]
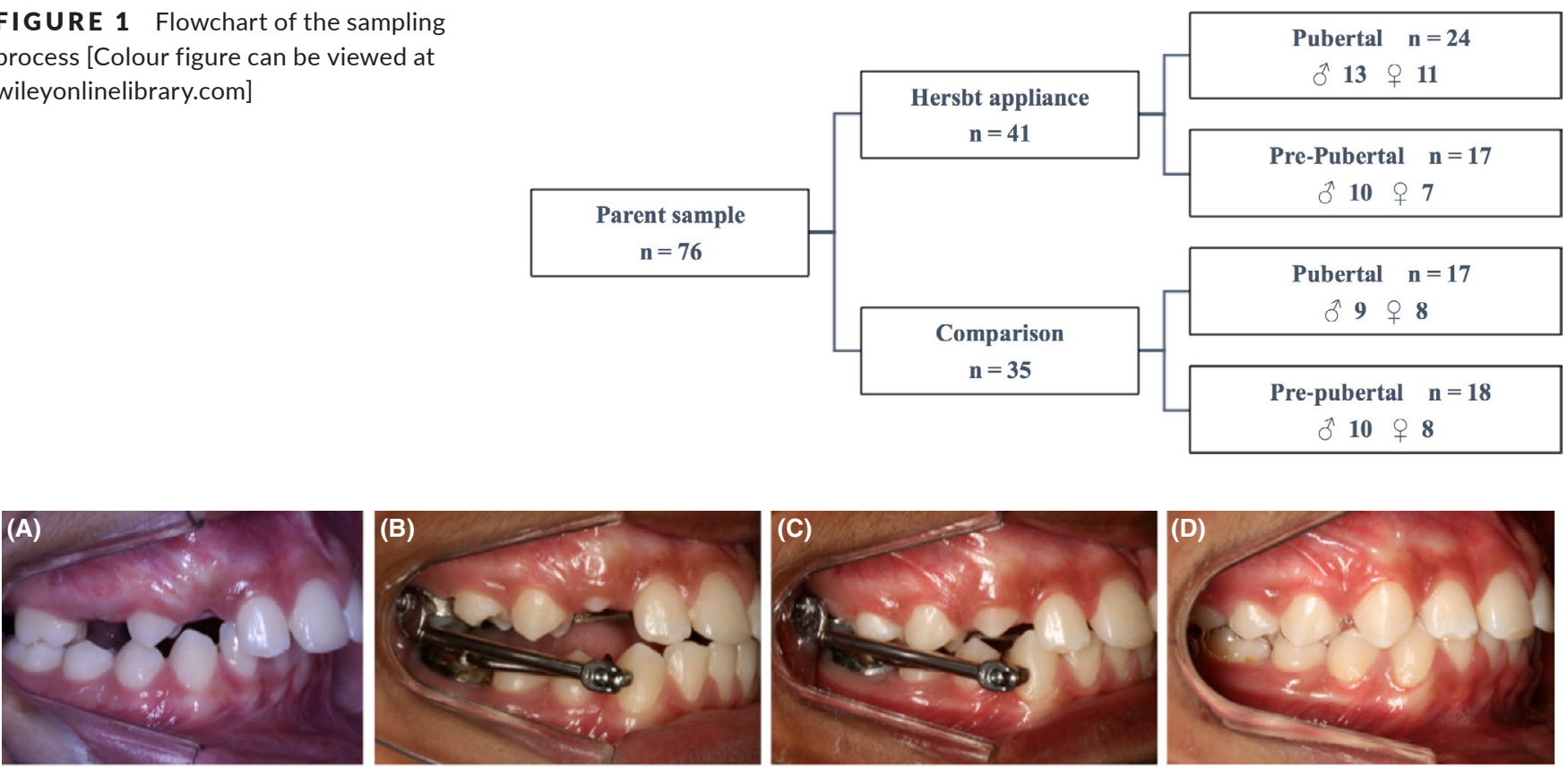

(E)

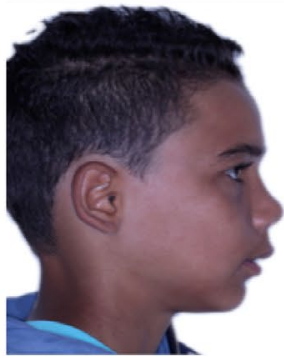

(F)

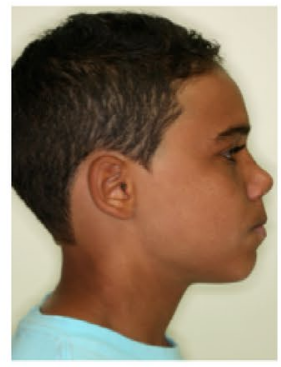

(G)

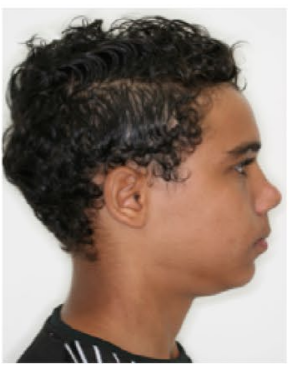

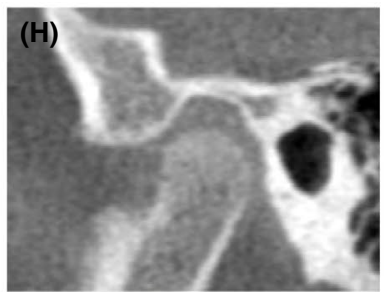
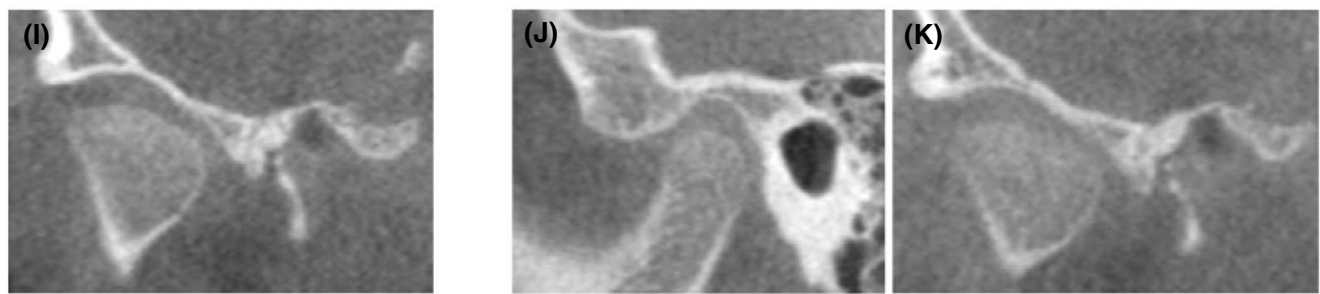

FIGURE 2 Herbst appliance treatment of a pre-pubertal patient. (A, E and H, I) Pre-treatment; (B and F) immediately after Herbst appliance insertion; (C) two months after Herbst appliance insertion; (D, G, J and K) the Herbst appliance was removed after eight months of treatment [Colour figure can be viewed at wileyonlinelibrary.com]

\section{2 | Image acquisition}

CBCTs were performed using the i-Cat machine (Imaging Sciences International, LLC) with isotropic $0.3 \times 0.3 \times 0.3 \mathrm{~mm}$ voxel, with the image obtained with the patient's teeth in maximum intercuspation. For the patients treated with the Herbst appliance, the CBCTs were taken before HA delivery (TO) and after 8 months (PHG) or 12 months (PPHG) of treatment (T1). HA patients had their T1 scans taken within two weeks after HA removal.

\section{3 | Image analysis}

The image analysis procedures were performed using ITK-SNAP (open-source software, www.itksnap.org) and 3D SLICER CMF (open-source software, www.slicer.org). The sequence included the following: (a) virtual 3D surface models construction; (b) head orientation of the TO scans in the same Cartesian coordinate system ${ }^{25}$; (c) manual approximation to achieve the best fit of T1 scans in TO pre-oriented scans, using the inner surface of the roof of the glenoid fossa roof as reference for independent right and left side superimposition; (d) automated voxel-based registration to improve the manual approximation, using as the region of interest for the volumetric superimposition the right and left side fossae, independently (Figure 3 shows the anatomic boundaries); and (e) quantitative and qualitative measurements.

To avoid bias in the identification of the landmarks, the pre-labelling of five skeletal landmarks in the TO oriented and T1 registered scans was performed simultaneously in multiplanar views 

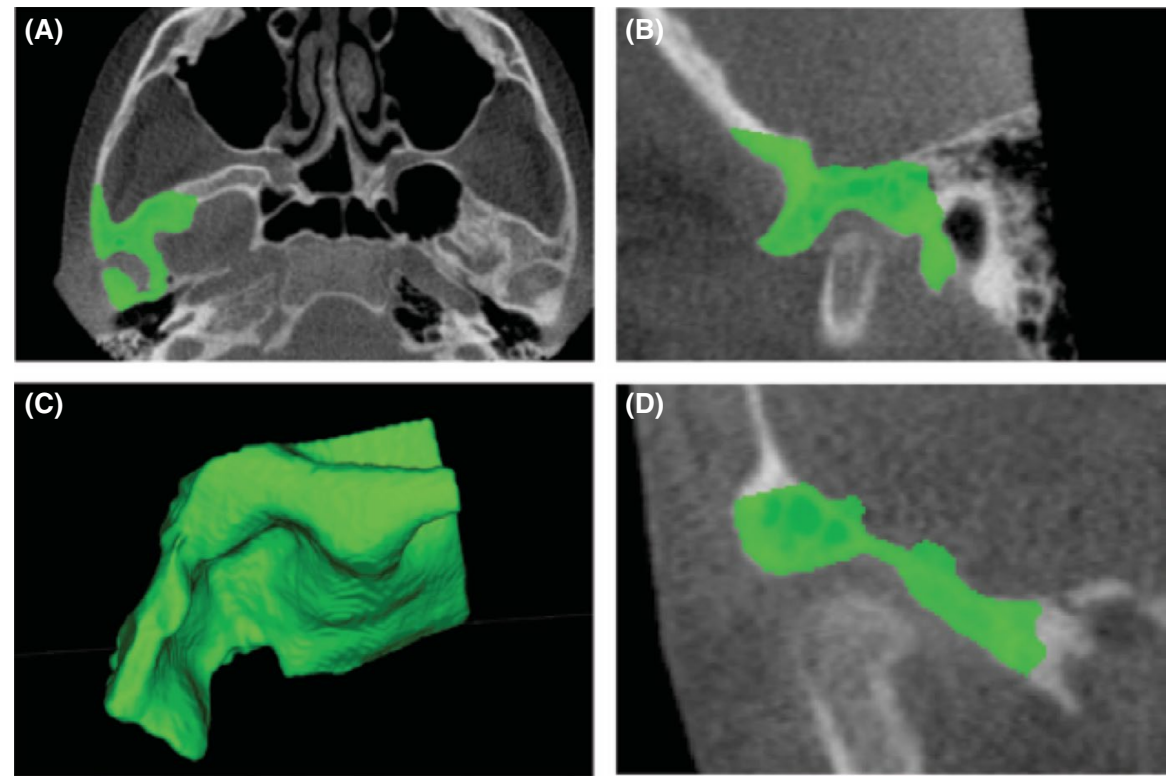

FIGURE 3 Label maps in the axial (A), sagittal (B) and coronal (D) views of the glenoid fossa and its $3 \mathrm{D}$ reconstruction (C). The boundaries of the anatomic region of interest used as the reference for the voxel-based regional registration are shown. Condylar positional and rotational changes from T0 to T1 were calculated relative to this region of reference. Right and left side volumetric superimpositions were carried out for independent assessment of the both sides condylar/ glenoid fossa relationship [Colour figure can be viewed at wileyonlinelibrary.com] (sagittal, axial and coronal; Figure 4), using two independent computer screens, one for T0 scan and the second screen for the T1 scan. Landmarks identified and plotted in planar views become a 3D sphere after volumetric reconstruction. The position of each landmark was verified further on the 3D models for accuracy.

The sagittal slice parallel to the mid-sagittal plane, passing through the geometric centre of the contour of the glenoid fossa roof in the axial view, was the reference for the standardized identification of landmarks 1, 2 and 3. The geometric centre of the roof of the glenoid fossae was identified in the first axial slice that showed the full roof of each glenoid fossa. To achieve it, the computer mouse scroll was run from top to bottom in the axial view. The roof of the glenoid fossa appears as a very thin layer of bone.

In the sagittal view (Figure 4A), three landmarks were identified: (1) the most superior point of the contour of the condyle; (2) the most anterior point of the contour of the condyle; (3) the most posterior point of the contour of the condyle. Translation of the condyles within the glenoid fossa was measured by the linear displacement of landmark 1 from T0 to T1. The pitch rotation was calculated with reference landmarks 2 and 3 (Figure 4A).

Landmarks 4 and 5 were located in the axial slice (Figure 4C) that showed the greatest medial-lateral dimension of the condyles as follows: (4) the most lateral point of the condyle and (5) the most medial point of the condyle. Landmarks 4 and 5 were used to calculate roll (Figure 4B) and yaw (Figure 4C).

Quantitative assessment of the changes in the position (translations and rotations) of the condyles from TO to $\mathrm{T} 1$ relative to their glenoid fossae was performed using 3D point-to-point measurements in the $\mathrm{X}, \mathrm{Y}, \mathrm{Z}$ projections and the Euclidean 3D distance, using the SLICER Q3DC tool. Qualitative visual analysis of the displacement of the condyle from T0 to T1 was performed using semi-transparent overlays and closest-point colour mapping (Figure 5), by way of Model-to-Model distance and Shape Population Viewer tools of SLICER software.
Dolphin Imaging software version 11.5 was used to reconstruct lateral cephalometric images (2D) from the DICOM files of the CBCT scan, and the CVM assessment was based on these 2D images.

\section{4 | Statistical analysis}

Data analysis was performed with SPSS software (version 21.0; Chicago, IL). The same operator (P.L.C.V.) performed the orientation of the scans, the volumetric superimposition and the measurements
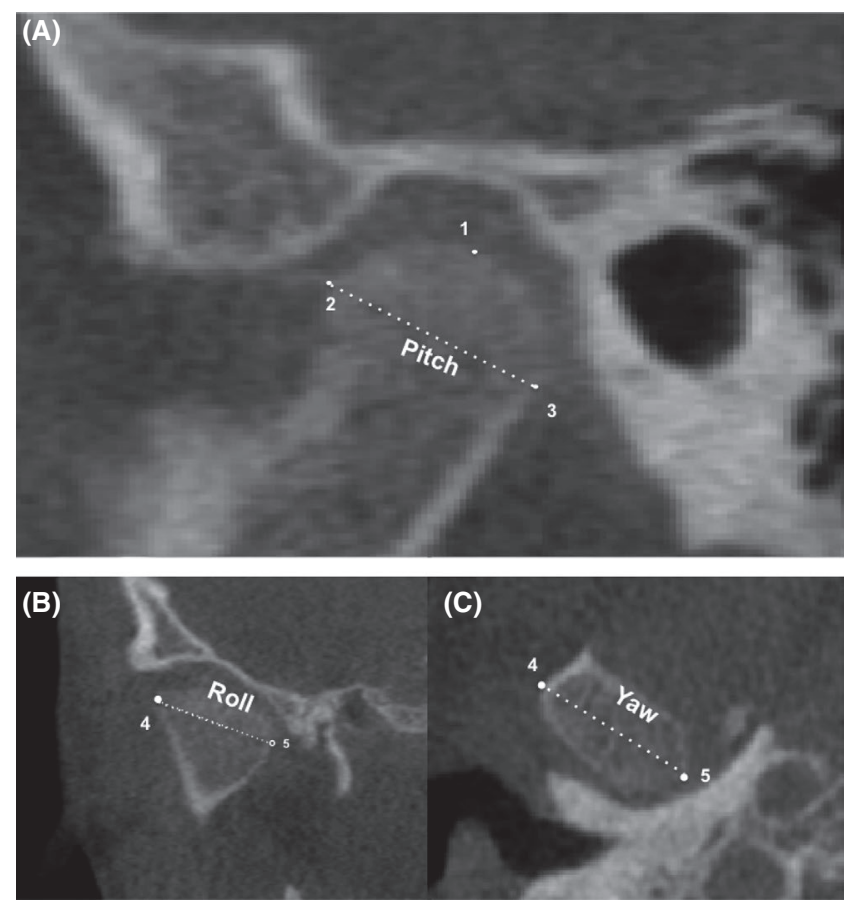

FIG URE 4 Landmarks were identified in the sagittal (A), coronal (B) and axial (C) views of the condyle. Landmarks 4 and 5 were plotted in the axial view and projected in coronal view 
FIGURE 5 Semi-transparent overlay shows solid model (TO) and black mesh model (T1) with minor pitch rotation (A), colour map allows visual comparison of changes from T0 to T1 in lateral and medial perspectives (B and C) [Colour figure can be viewed at wileyonlinelibrary. com]

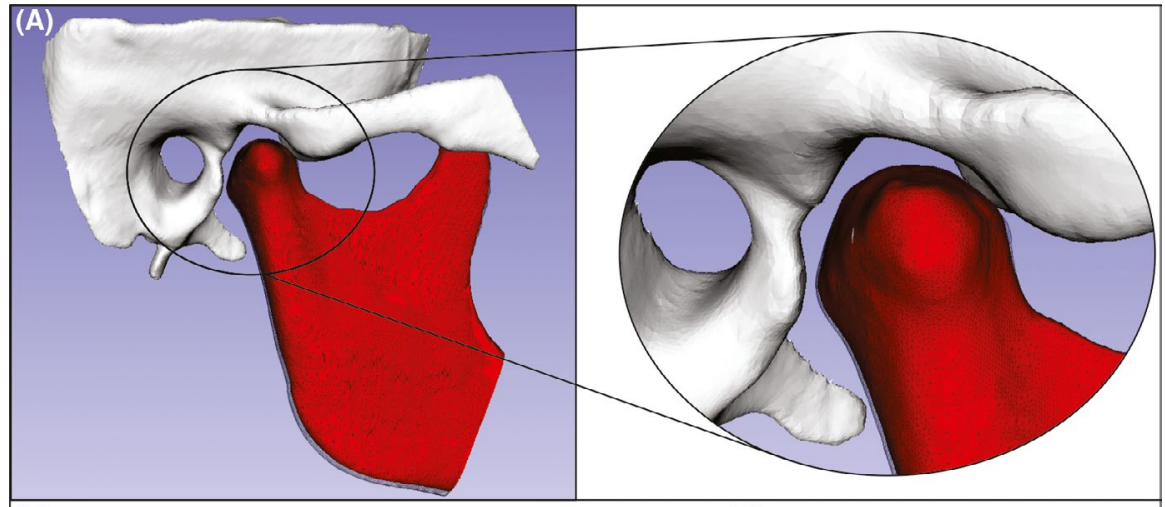

(B)

1

(C)

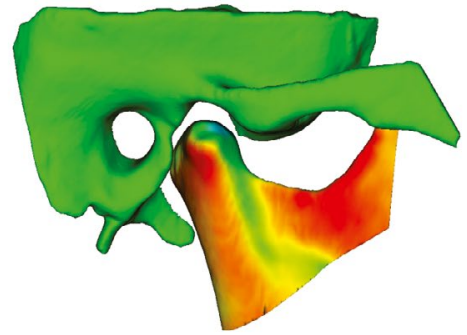

0.5

0

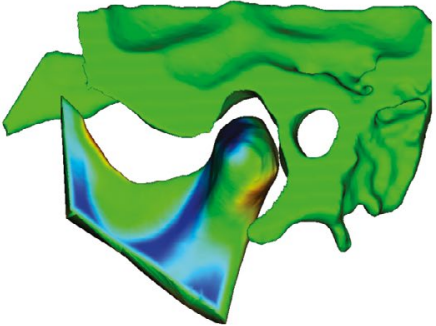

TAB LE 1 Descriptive data of the displacements $(\mathrm{mm})$ and rotations (degree) of the condyles from its original position within the glenoid fossa (T1-TO) and the comparison among the four groups

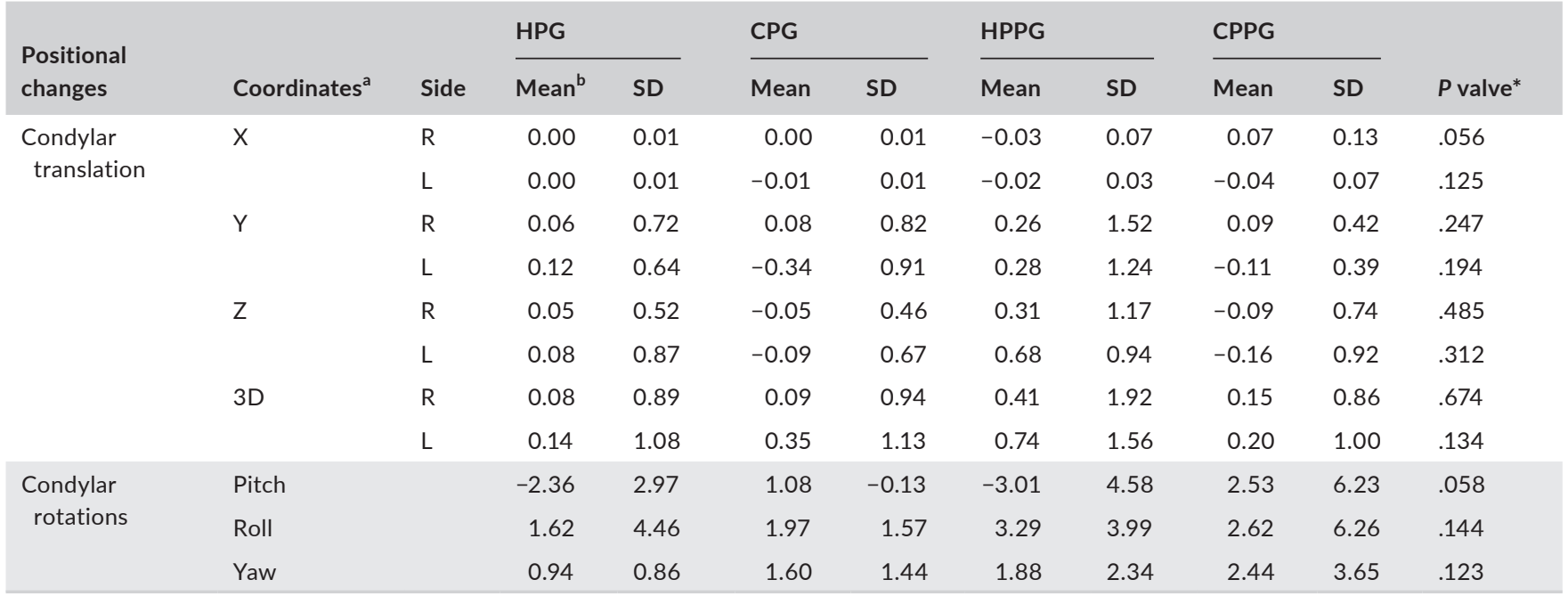

Abbreviations: CPG, comparison pubertal group; CPPG, comparison pre-pubertal group; HPG, Herbst pubertal group; HPPG, Herbst pre-pubertal group.

${ }^{a} \mathrm{X}$, mesial-lateral; $\mathrm{Y}$, anterior-posterior; Z, superior-inferior; 3D, Euclidian distance.

b(+), Rightward; forward; upward; clockwise rotation; (-), Leftward; backward; downward; counterclockwise rotation.

${ }^{*} P$ value calculated with Kruskal-Wallis test.

after three months of training with the senior investigators. Repetition of all steps for 30 patients was carried out at least three months after the first analysis to test intrarater consistency and agreement. A second operator (L.O.F.) also calibrated by the senior author performed all steps for 30 patients, independently, to test inter-rater agreement.
For the continuous variables, both intra-observer and interobserver agreement measurements were tested using intraclass correlation coefficients (ICC), with a confidence level of $95 \%$. Intrarater agreement for CVM method was tested with weighted kappa. Random error was measured according to Dahlberg's 


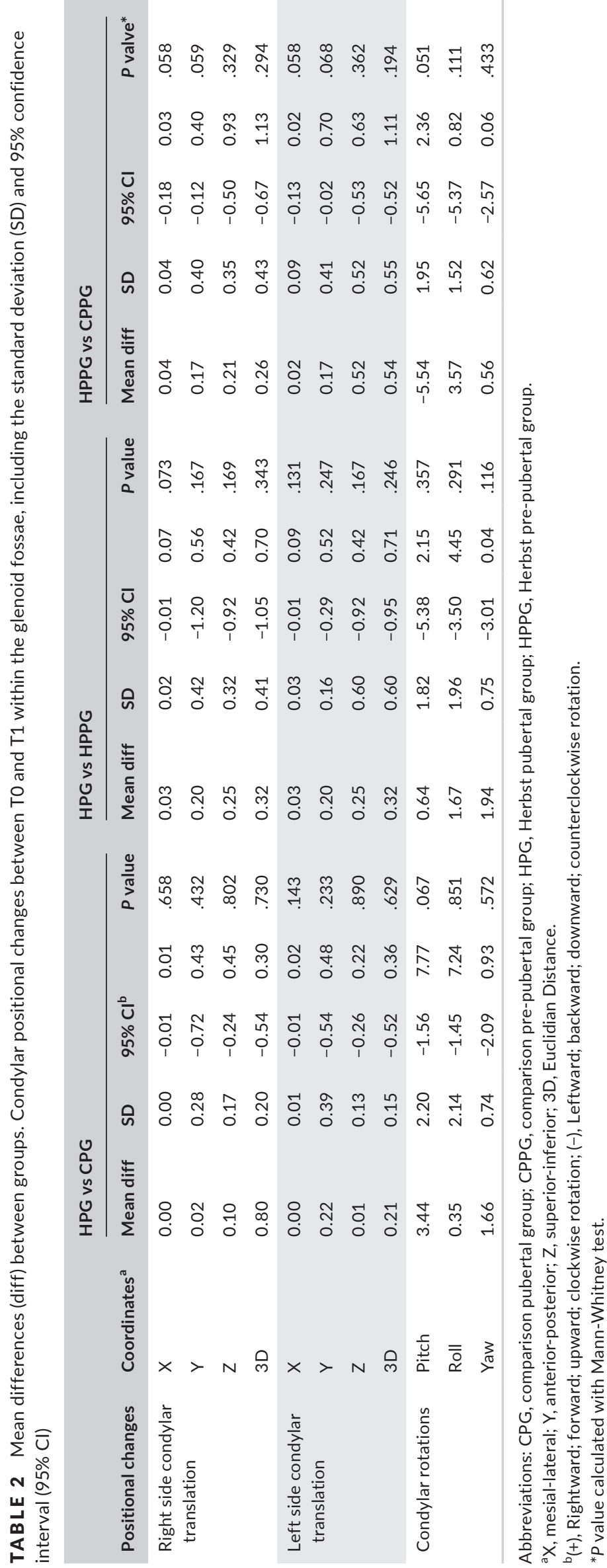


formula. ${ }^{26}$ Systematic error (bias) was assessed using the paired t test.

The comparison of the mean age between groups was performed with Student's $t$ test. Comparison of the distribution of gender was run with chi-square test. Some variables did not show normal distribution, and thus, the non-parametric Kruskal-Wallis test was used to compare differences among the four groups (Table 1). The MannWhitney test was used to compare differences between two independent groups (Table 2). No missing data were generated as results. The level of significance was set at $5 \%$.

\section{3 | RESULTS}

Excellent agreement for the CVM classification was found (>0.92). The ICCs were greater than 0.81 for both intra-observer and interobserver repeated measurements. There were no statistically significant systematic errors between the two measurements performed by the same operator $(P>.05)$, and random error values varied between $0.45 \mathrm{~mm}$ and $0.77 \mathrm{~mm}$.

From the records of 1328 consecutive patients treated at the two Graduate Programs in Orthodontics, 1134 were excluded because they did not present a Class II malocclusion or were not in pre-pubertal or pubertal stages of maturation. ${ }^{23}$ Thus, 194 individuals were potentially eligible for the current study. Other modalities of Class II correction or the absence of CBCT scans determined the exclusion of 118 treated patients. Thus, the records of 76 patients were included in the current study. As the study participants belong to similar demographic, clinical and social conditions, the presence of potential confounders was minimized.

No difference in the distribution of patients according to the mean age of groups (PPHG vs PPCG, $P=.198$; PHG vs PCG, $P=.268$ ) was found. Also, no difference in the distribution of patients according to gender was detected (PPHG vs PPCG, $P=.845$; PHG vs PCG, $P=$.938).

Table 1 shows the descriptive data, including the mean and standard deviation for the condylar translation and rotations within the glenoid fossa of treated and comparison groups, during the pubertal and pre-pubertal stages of maturation. The positional changes in the condyles from T0 to T1 within the glenoid fossae were smaller than $0.75 \mathrm{~mm}$ and were not statistically significant difference $(P>.05)$.

The HA group presented clockwise pitch, while the comparison groups showed counterclockwise pitch. However, the pitch change was small ( $<3$ degrees) in both the treated and non-treated groups during the pre-pubertal and pubertal stages of maturation, presenting no statistically significant differences. Roll and yaw also were small and not statistically significant different.

The mean differences between treated and non-treated patients in the two stages of maturation, including the $95 \%$ confidence interval, are compared in Table 2. No statistically significant differences were found in the pattern of the changes from TO to T1, both in the pre-pubertal and pubertal individuals, in the HA and comparison groups.

\section{4 | DISCUSSION}

The results of the present study indicated that regardless of the stage of maturation and the length of treatment (8 or 12 months), patients in pre-pubertal and pubertal maturational stages reestablish their original TMJ position within the glenoid fossa by the end of Herbst appliance treatment. While significant condylar growth might not be achievable with early Herbst treatment, ${ }^{1,2}$ it is important for clinicians to know whether early treatment of Class II malocclusion is indicated for psychosocial problems or increased risk of incisor trauma. The concern that the condyles will be displaced into a pathological position is not supported by the data generated in this study.

Using a novel 3D imaging methodology, our findings corroborate previous $2 \mathrm{D}$ and $3 \mathrm{D}$ reports that effective treatment of Class II malocclusion adolescent patients using dentofacial orthopaedics does not change the position of the condyles relative to the glenoid fossa. Ruf and Pancherz ${ }^{7}$ analysed MRI images of 15 Class II patients (initial ages 11.5 to 17.5 years) treated with HA for 7 months. They reported 'the condyle-fossa relationship was, on average unaffected by Herbst therapy'. Kinzinger et $\mathrm{al}^{16}$ and Kinzinger et $\mathrm{al}^{17}$ evaluated MRI's of 20 Class II patients (mean starting age 15.6 years, ranging from 12 to 25.9 years) treated with a fixed mandibular advancement device. They found that 'improved dental occlusion was not achieved at the price of a change to an unphysiological position in the temporomandibular joints'. Recently, Atresh et $\mathrm{al}^{19}$ reported in adolescents 12 to 13 years of age at the beginning of treatment that there were no significant differences in the 3D positions of the geometric centre of the condyle after orthodontic treatment had been completed, with the overall trend resulting a slightly posterior displacement of both glenoid fossae and condyles.

With regard to Herbst appliance treatment, previous reports mostly had used 2D assessments and measurements of condylar spatial changes. ${ }^{7-17}$ The current study utilized 3D assessment of five landmarks located around the condyle, along with visual analytics tools such as semi-transparent overlays of $3 D$ models and colour mappings to provide a qualitative and visual tool to validate the objective measurements. In addition, this study used regional volumetric superimposition of each glenoid fossa to avoid the problems associated with transverse craniofacial growth, which may impact the assessment of changes in bilateral facial structures negatively, with 2D superimpositions of mid-sagittal structures.

During the first two decades of life, the TMJ region and the posterior region of the cranial base undergo three-dimensional changes associated with normal growth and development. Significant transverse gain occurs in the TMJ to compensate for the $\mathrm{V}$-shaped growth of the mandible. ${ }^{27}$ Figure 5 suggests bone apposition in the lateral surfaces (red colour) and bone resorption in the medial aspect (blue colour), but the condyles kept their original position within the glenoid fossa (green colour). The maintenance of condylar position within the glenoid fossa can also be seen in Figure $2 \mathrm{H}-\mathrm{K}$.

Bone remodelling in the superior and posterior regions of the condyle $^{28}$ and in the inner surface of the fossa ${ }^{29}$ may have been 
one of the factors that contributed to the reestablishment of the condyle-fossa relationship during HA treatment; however, with the current study methodology, this type of bone remodelling cannot be measured.

3D studies have suggested compensatory TMJ bone growth resulting from Herbst treatment. LeCornu et $a^{18}$ showed significant bone remodelling activity within the glenoid fossae of HA patients. Souki et $\mathrm{al}^{30}$ found a significant increase and change in the pattern of bone remodelling and a different magnitude and direction of condylar growth after HA treatment. However, bone remodelling processes, both from the condyles and/or the glenoid fossae, cannot explain the extensive drift and repositioning of the condyle within the glenoid fossa after 8 or 12 months of treatment, following mandibular advancements ranging from 4 to $10 \mathrm{~mm}$.

The reestablishment of the original condyle-fossa relationship, after HA treatment, is likely due to a combination of minor bone remodelling changes as well as the mechanical drift of the condyle into its original position due to soft tissues traction. Huang and Ross ${ }^{31}$ concluded that masticatory muscles as well as the other stretched connective tissues surrounding the mandible have been associated with the pull of the condyle back into the fossa after surgical advancement of the retrognathic mandible in growing children. Voudouris and Kuftine ${ }^{32}$ have shown that viscoelastic components of the TMJ (retrodiscal fibrous force, fibrous capsule force and synovial fluid perfusion) exert a pull back traction at the condylar head after mandibular advancement with orthopaedic appliances.

The rebound effect forced by the soft tissues might play a significant role in the backward and upward drift of the mandible during the months following HA insertion. Soft tissues including the articular capsule, articular ligaments and lateral pterygoid muscles are attached around the head of the condyle and play an important role in the movement of the head, the mandible and the articular disc.

The TMJ musculature, however, has been studied more than other soft tissues. Animal studies have shown cellular, structural and functionality adaptive changes in the lateral pterygoid muscle after protrusion of the mandible. ${ }^{33,34}$ These change not only stimulate bone remodelling ${ }^{31}$ but also might help to achieve the homeostasis of the TMJ. Clinicians should be aware that the mandibular forward positioning obtained the day of HA insertion will not be maintained during the treatment, and the excessive prognathic profile generated in the day of the HA insertion will reduce during the following weeks. Pancherz ${ }^{35}$ have suggested building the construction bite taking in an overcorrected edge-to-edge incisor position. This recommendation takes in account the rebound effect of the mandible.

The major limitation of this study was the retrospective design. Additional weaknesses of the sample include differences in the observation times between pre-pubertal and pubertal Herbst patients and the variability of the degree of severity of the Class II skeletal malocclusions among patients. Sample size calculation was based on an effect size of 1.35 with a SD of 0.74 and acceptable difference between groups of $1 \mathrm{~mm}$. Thus, the current absence of difference between groups must be analysed taking into consideration this arbitrarily given clinically acceptable distance difference between groups. If a smaller clinical tolerance were considered, a smaller effect size would recommend a significantly greater amount of patients, which due to the retrospective design of the current investigation would not be feasible. Therefore, the current findings need to be interpreted with caution, despite that our findings are very similar to previously reported data, ${ }^{7,16-19}$ suggesting the generalizability (external validity) of the study results. A prospective randomized clinical trial would compensate for such weaknesses, and however, due to the current ALARA principle it would not be ethical to run prospective studies with $\mathrm{CBCT}$ in children. The current retrospective investigation was carried on records previously collected when another imaging protocol was used. The two departments whose database was searched have nowadays a more conservative approach and do not acquire CBCT scans for every single orthodontic patient.

The findings of the present study as well as those from previous investigations on this topic suggest that clinicians should not be concerned with the anterior displacement of condyles immediately after HA insertion, regardless if the patient is in the pre-pubertal or pubertal stage of growth. The rebound effect associated with mandibular growth is likely to reestablish normal fossa/condyle relationship, and at the end of the treatment, the condyles will be seated in their original position.

\section{5 | CONCLUSIONS}

Herbst appliance treatment did not change the original condylefossa relationship at the time of HA removal, regardless the stage of skeletal maturation. The condyles remained spatially stable relative to their glenoid fossae after 8 or 12 months of treatment.

\section{ACKNOWLEDGEMENTS}

We thank Luana Araujo for her technical contribution in the data collection and technical support. This study was partially funded by CAPES (Coordenação de Aperfeiçoamento de Pessoal de Nível Superior, Grant \#88881-134753/2016-01). The investigation was approved by the Institutional Review Board of the Pontifical Catholic University of Minas Gerais (number 057992/2016).

\section{CONFLICT OF INTEREST}

Dr. Cheib Vilefort received grants from the Brazilian National Council for Research to perform the current 3D analysis at the University of Michigan. Other authors declare that they have no conflict of interest.

\section{ORCID}

Tung Nguyen (iD https://orcid.org/0000-0002-4978-3558

Lorenzo Franchi (iD https://orcid.org/0000-0002-2072-460X

Bernardo Quiroga Souki (iD https://orcid.org/0000-0002-1907-9519 


\section{REFERENCES}

1. Perinetti G, Primožič J, Furlani G, Franchi L, Contardo L. Treatment effects of fixed functional appliances alone or in combination with multibracket appliances: A systematic review and meta-analysis. Angle Orthod. 2015;85:480-492.

2. Pavoni C, Lombardo EC, Lione R, et al. Treatment timing for functional jaw orthopaedics followed by fixed appliances: a controlled long-term study. Eur J Orthod. 2018;40:430-436.

3. Batista KB, Thiruvenkatachari B, Harrison JE, O'Brien KD. Orthodontic treatment for prominent upper front teeth (Class II malocclusion) in children and adolescents. Cochrane Database Syst Rev. 2018;13(3):CD003452.

4. O'Brien K, Wright J, Conboy F, et al. Effectiveness of early orthodontic treatment with the Twin-block appliance: a multicenter, randomized, controlled trial. Part 2: Psychosocial effects. Am J Orthod Dentofacial Orthop. 2003;124:488-494.

5. Cacho A, Ono T, Kuboki T, Martin C. Changes in joint space dimension after the correction of Class II division 1 malocclusion. Eur J Orthod. 2015;37:1-7.

6. Chavan SJ, Bhad WA, Doshi UH. Comparison of temporomandibular joint changes in Twin Block and Bionator appliance therapy: a magnetic resonance imaging study. Prog Orthod. 2014;15:57.

7. Ruf S, Pancherz H. Temporomandibular joint growth adaptation in Herbst treatment: a prospective magnetic resonance imaging and cephalometric roentgenographic study. Eur J Orthod. 1998;20:375-388.

8. Pancherz H, Ruf S, Kohlhas P. "Effective condylar growth" and chin position changes in Herbst treatment: a cephalometric roentgenographic long-term study. Am J Orthod Dentofacial Orthop. 1998;114:437-446.

9. Pancherz H, Fischer S. Amount and direction of temporomandibular joint growth changes in Herbst treatment: a cephalometric longterm investigation. Angle Orthod. 2003;73:493-501.

10. Serbesis-Tsarudis C, Pancherz H. "Effective" TMJ and chin position changes in Class II treatment. Angle Orthod. 2008;78:813-818.

11. Ruf $\mathrm{S}$, Pancherz $\mathrm{H}$. Temporomandibular joint remodeling in adolescents and young adults during Herbst treatment: A prospective longitudinal magnetic resonance imaging and cephalometric radiographic investigation. Am J Orthod Dentofacial Orthop. 1999;115:607-618.

12. Ruf $\mathrm{S}$, Pancherz H. Does bite-jumping damage the TMJ? A prospective longitudinal clinical and MRI study of Herbst patients. Angle Orthod. 2000;70:183-199.

13. VanLaecken R, Martin CA, Dischinger T, Razmus T, Ngan P. Treatment effects of the edgewise Herbst appliance: a cephalometric and tomographic investigation. Am J Orthod Dentofacial Orthop. 2006;130:582-593.

14. Popowich K, Nebbe B, Major PW. Effect of Herbst treatment on temporomandibular joint morphology: a systematic literature review. Am J Orthod Dentofacial Orthop. 2003;123:388-394.

15. Aidar L, Abrahão M, Yamashita HK, Dominguez GC. Herbst appliance therapy and temporomandibular joint disc position: a prospective longitudinal magnetic resonance imaging study. Am J Orthod Dentofacial Orthop. 2006;129:486-496.

16. Kinzinger G, Roth A, Gülden N, Bücker A, Diedrich PR. Effects of orthodontic treatment with fixed functional orthopaedic appliances on the condyle-fossa relationship in the temporomandibular joint: a magnetic resonance imaging study (Part I). Dentomaxillofac Radiol. 2006;35:339-346.

17. Kinzinger G, Kober C, Diedrich P. Topography and morphology of the mandibular condyle during fixed functional orthopedic treatment - a magnetic resonance imaging study. J Orofac Orthop. 2007;68:124-147.

18. LeCornu M, Cevidanes L, Zhu H, Wu C-D, Larson B, Nguyen T. Three-dimensional treatment outcomes in Class II patients treated with the Herbst appliance: a pilot study. Am J Orthod Dentofacial Orthop. 2013;144:818-830.
19. Atresh A, Cevidanes L, Yatabe M, et al. Three-dimensional treatment outcomes in Class II patients with different vertical facial patterns treated with the Herbst appliance. Am J Orthod Dentofac Orthop. 2018;154(2):238-248.e1.

20. Al-Saleh M, Alsufyani N, Flores-Mir C, Nebbe B, Major PW. Changes in temporomandibular joint morphology in class II patients treated with fixed mandibular repositioning and evaluated through 3D imaging: a systematic review. Orthod Craniofac Res. 2015;18:185-201.

21. von Elm E, Altman DG, Egger M, et al. The strengthening the reporting of observational studies in epidemiology (STROBE) statement: guidelines for reporting observational studies. J Clin Epidemiol. 2008;61:344-349.

22. Arieta-Miranda JM, Silva-Valencia M, Flores-Mir C, ParedesSampen NA, Arriola-Guillen LE. Spatial analysis of condyle position according to sagittal skeletal relationship, assessed by cone beam computed tomography. Prog Orthod. 2013;14(1):36.

23. McNamara JA Jr, Franchi L. The cervical vertebral maturation method: A user's guide. Angle Orthod. 2018;88:133-143.

24. Riolo ML, Moyers RE, McNamara JA Jr, Hunter WS. An atlas of craniofacial growth: Cephalometric standards from The University School Growth Study. The University of Michigan. Monograph 2, Craniofacial Growth Series, Center for Human Growth and Development. The University of Michigan, Ann Arbor, 379 pages, 1974.

25. Ruellas AC, Tonello C, Gomes LR, et al. Common 3-dimensional coordinate system for assessment of directional changes. Am J Orthod Dentofac Orthop. 2016;149:645-656.

26. Houston WJ. The analysis of errors in orthodontic measurements. Am J Orthod. 1983;83:382-390.

27. Buschang PH, Santos-Pinto A. Condylar growth and glenoid fossa displacement during childhood and adolescence. Am J Orthod Dentofacial Orthop. 1998;113:437-442.

28. Franchi L, Pavoni C, Faltin K, Bigliazzi R, Gazzani F, Cozza P. Thinplate spline analysis of mandibular shape changes induced by functional appliances in Class II malocclusion : A long-term evaluation. J Orofac Orthop. 2016;77(5):325-333.

29. Woodside DG, Metaxas A, Altuna G. The influence of functional appliance therapy on glenoid fossa remodeling. Am J Orthod Dentofacial Orthop. 1987;92(3):181-198.

30. Souki BQ, Vilefort P, Oliveira DD, et al. Three-dimensional skeletal mandibular changes associated with Herbst appliance treatment. Orthod Craniofac Res. 2017;20:111-118.

31. Huang CS, Ross RB. Surgical advancement of the retrognathic mandible in growing children. Am J Orthod. 1982;82:89-103.

32. Voudouris JC, Kuftinec MM. Improved clinical use of Twin-block and Herbst as a result of radiating viscoelastic tissue forces on the condyle and fossa in treatment and long-term retention: growth relativity. Am J Orthod Dentofacial Orthop. 2000;117:247-266.

33. Easton JW, Carlson DS. Adaptation of the lateral pterygoid and superficial masseter muscles to mandibular protrusion in the rat. Am J Orthod Dentofac Orthop. 1990;97:149-158.

34. McNamara JA Jr, Carlson DS. Quantitative analysis of temporomandibular joint adaptations to protrusive function. Am J Orthod. 1979;76:593-611.

35. Pancherz H. The Herbst appliance - Its biologic effects and clinical use. Am J Orthod. 1985;87:1-20.

\section{How to cite this article: Cheib Vilefort PL, Farah LO,}

Gontijo HP, et al. Condyle-glenoid fossa relationship after Herbst appliance treatment during two stages of craniofacial skeletal maturation: A retrospective study. Orthod Craniofac Res. 2019;22:345-353. https://doi.org/10.1111/ocr.12338 